\title{
Physiological and clinical effects of diurnal noninvasive ventilation in hypercapnic
} COPD

\author{
O. Díaz*, P. Bégin\#, M. Andresen`, M.E. Prieto*, C. Castillo*, \\ J. Jorquera* and C. Lisboa*
}

ABSTRACT: To assess the clinical impact of noninvasive mechanical ventilation (NIMV) on stable hypercapnic chronic obstructive pulmonary disease, changes in exercise capacity, dyspnoea and simple physiological parameters were evaluated. The time course of these effects during treatment and recovery was also assessed.

Patients were randomly allocated to NIMV $(n=27)$ or sham-NIMV $(n=15)$, applied $3 h \cdot$ day $^{-1}, 5$ days a week, for 3 weeks. A 6-min walking distance (6MWD), arterial blood gases, spirometry, pattern of breathing, mouth occlusion pressure $\left(P_{0.1}\right)$, and respiratory system impedance $\left(P_{0.1} /\right.$ tidal volume $(V T)$ /inspiratory time $(t I))$ were measured weekly during treatment and 2 weekly during follow-up. Transition dyspnoea index (TDI) was also measured.

During NIMV, carbon dioxide arterial tension decreased progressively, concomitantly with a slow deep pattern of breathing, a proportional increase in the forced expiratory volume in one

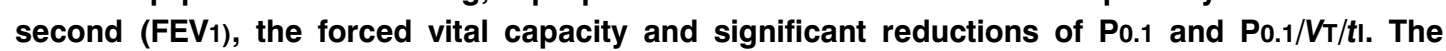
6MWD improved by a mean of $76 \mathrm{~m}$ after NIMV, and by $73 \mathrm{~m}$ and $61 \mathrm{~m} 1$ and 2 weeks, respectively, after treatment. Dyspnoea improved with a mean TDI of three points. Changes in 6MWD were highly related to TDI and to a lesser extent to changes in FEV $1(r=0.60)$.

The current authors conclude that noninvasive mechanical ventilation has significant and sustained clinical impact in stable hypercapnic chronic obstructive pulmonary disease.

KEYWORDS: Chronic obstructive pulmonary disease, dyspnoea, exercise capacity, hypercapnia, noninvasive ventilation

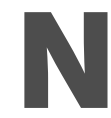

oninvasive mechanical ventilation (NIMV) has proven useful in treating patients with severe chronic obstructive pulmonary disease (COPD) and acute respiratory failure (ARS) [1]. However, reports on its usefulness in stable COPD are controversial [2]. In a previous study, the current authors have shown that intermittent diurnal NIMV reduces hypercapnia and hypoxaemia in patients with severe COPD [3]. The mechanisms underlying these changes were a combination of lung deflation, reduction of respiratory loads, and changes in ventilatory pattern. The current findings were in line with a previous uncontrolled study [4]. The rationale of this ventilation strategy was primarily based on the hypothesis that high inflation pressure is a key factor for success [5] and that it could be better achieved with closer supervision in the awake patient.

In order to establish if the physiological benefits previously reported with this new ventilatory strategy [3] are accompanied by concomitant and sustained clinical improvements, a randomised placebo-controlled study of weekly NIMV effects on physiology, dyspnoea and exercise capacity in COPD patients with stable hypercapnic respiratory failure was carried out. In addition, residual effects of NIMV during 2 weeks after the end of treatment was also explored.

\section{METHODS}

\section{Patients}

A total of 62 consecutive patients under chronic care at the Universidad Católica de Chile (Santiago, Chile) outpatient clinic for COPD [6], whose resting daytime carbon dioxide arterial tension $\left(\mathrm{Pa}_{\mathrm{a}} \mathrm{CO}_{2}\right)$ was $\geqslant 6.65 \mathrm{kPa}(50 \mathrm{mmHg})$, arterial oxygen tension $\left(\mathrm{Pa}_{\mathrm{a}} \mathrm{O}_{2}\right)<7.98 \mathrm{kPa}$ $(60 \mathrm{mmHg})$, and who were in a clinically and functionally stable state were evaluated. A total of 12 patients were excluded from the study: four with $\geqslant 10 \%$ increase in per cent predicted forced expiratory volume in $1 \mathrm{~s}$ (FEV1) after inhaled salbutamol, three with obstructive sleep apnoeas, two with clinical exacerbations during the

\section{AFFILIATIONS}

*Dept of Respiratory Diseases and

"Dept of Medicine, Universidad

Católica de Chile, Santiago, Chile.

\#Respiratory Division, Complexe

hospitalier de la Sagamie,

Chicoutimi, Dept of Medicine,

University of Montreal, Canada.

CORRESPONDENCE

0. Díaz

Dept of Respiratory Diseases

Universidad Católica de Chile

Marcoleta 345

Piso 4. Santiago

Chile

Fax: 5626335255

E-mail: diazp@rdc.cl

Received:

March 222005

Accepted after revision:

August 112005

SUPPORT STATEMENT

This work was supported by Grant \# 1030652 from Fondo Nacional de Ciencias y Tecnología (Fondecyt), Chile.

Online ISSN 1399-3003 
preceding 4 weeks, and three with other concomitant disorders (left ventricular failure, peripheral vascular occlusive disease, orthopaedic disorder). Fifty patients agreed to participate in the study, which was approved by the Ethics Committee of Universidad Católica de Chile. A written informed consent was obtained from the patients before enrolment into the study. All patients had stopped smoking for $\geqslant 1 \mathrm{yr}$ prior to the study and were on long-term oxygen therapy. None were participating in a respiratory training programme, receiving home mechanical ventilation, nor had previous exposure to NIMV.

\section{Study design}

A two-armed single-blinded randomised study of active (NIMV) versus sham-NIMV (s-NIMV) was conducted, with odds of 2:1 to receive NIMV, to add precision to the timecourse estimates of mean NIMV effects. Since s-NIMV and NIMV were performed in two separate rooms, patients from different groups were not in contact with each other, and thus remained blinded to the randomisation. Their treating physicians and the nurse supervising dyspnoea and exercise tolerance tests were also blinded to the type of treatment.

Patients first entered a 2-week run-in period, using their current medication, during which they were familiarised with measurements. Spirometry, arterial blood gases and clinical status were assessed at the time of recruitment and at the end of the run-in period. This was followed by a 3week study period, during which they were randomly allocated to NIMV or s-NIMV using a table of random numbers and sealed envelopes. Ventilation was applied under direct supervision by an experienced, registered nurse at the pulmonary function laboratory, on an outpatient basis. After the study period, patients were followed up for a 2-week period.

NIMV was delivered through a silicone face mask (HansRudolph, Kansas City, MO, USA) and a nonrebreathing valve via a bilevel positive airways pressure (BiPAP) ventilator in spontaneous mode (model S/T-D; Respironics, Inc., Murrysville, Pennsylvania, USA) and s-NIMV via a Siemens Servo ventilator 900C (Siemens; Solna, Sweden) in continuous positive airways pressure (CPAP) mode. Humidifiers were not used. NIMV and s-NIMV were applied for $3 \mathrm{~h} \cdot \mathrm{day}^{-1}, 5$ days a week, and during 3 weeks, as previously described [3]. In the NIMV group, the level of inspiratory pressure (IPAP) was gradually increased from $8 \mathrm{cmH}_{2} \mathrm{O}$ by $2 \mathrm{cmH}_{2} \mathrm{O}$ steps during the first hour of ventilation to the highest tolerated level. The latter was considered the target IPAP for that particular patient for the rest of the study. In most cases a gradual increase in pressure to the target IPAP was required at the beginning of each session to maintain the patient's comfort. The mean level of IPAP used was $18 \pm 2 \mathrm{cmH}_{2} \mathrm{O}$, whereas the expiratory positive airway pressure was set at a minimum $\left(2 \mathrm{cmH}_{2} \mathrm{O}\right)$. In the s-NIMV group, a low CPAP level of $2 \mathrm{cmH}_{2} \mathrm{O}$ was used. The current authors had previously shown that such low levels are not associated with an increase in inspiratory load [3]. The protocol allowed administration of supplemental $\mathrm{O}_{2}$ in variable concentrations for both groups in order to maintain an arterial oxygen saturation $\left(\mathrm{Sa}_{2} \mathrm{O}_{2}\right)$ near $94 \%$ during the ventilation sessions. Only two patients in the NIMV group needed supplemental oxygen to achieve the target $\mathrm{Sa}_{2} \mathrm{O}_{2}$, whereas all controls had to be supplemented with a mean inspiratory oxygen fraction of $0.24 \pm 0.03$.

\section{Measurements}

Measurements were made at baseline, i.e. after the run-in period, and at the end of each week during the treatment period, $24 \mathrm{~h}$ post the last session of mechanical ventilation, except for changes in resting dyspnoea which was measured only at the end of the treatment period. Follow-up assessments were performed 7 and 14 days after the end of treatment.

\section{Spirometry and arterial blood gases}

Subjects were studied sitting upright in a comfortable chair breathing room air, $\sim 4 \mathrm{~h}$ after inhalation of their usual bronchodilating medications. Spirometry was performed with a calibrated dry spirometer (Vitalograph ${ }_{\mathbb{R}}$; London, UK) according to the American Thoracic Society standards [7]. Reference values where those of KNUDSON et al. [8]. Resting daytime arterial blood gases (ABG) were measured with a Ciba Corning 238 gas analyzer (Ciba Corning Diagnostic Corp; Medfield, MA, USA).

\section{Breathing pattern, inspiratory muscle function and central respiratory drive}

Airflow was measured with a heated Hans Rudolph pneumotachograph (model 3700A: Raytech Instruments; Vancouver, British Columbia, Canada) and a differential pressure transducer (DP55 $\pm 3 \mathrm{cmH}_{2} \mathrm{O}$; Raytech Instruments). Volume was obtained by numerical integration of the flow signal. Tidal volume $(V \mathrm{~T})$, inspiratory time $(t \mathrm{I})$ and expiratory time $(t \mathrm{E})$, total breath duration and the mean inspiratory flow $(V \mathrm{~T} / t \mathrm{I})$ were obtained as average values from 1-min flow and volume records. Pressure at airway opening $(\mathrm{Pa}, \mathrm{o})$ was measured via a differential pressure transducer (DP55 $\pm 100 \mathrm{cmH}_{2} \mathrm{O}$; Raytech Instruments). Pressure and flow signals were amplified (AC Bridge amplifier-ACB module; Raytech Instruments) and sampled simultaneously at a rate of $100 \mathrm{~Hz}$ by a 16-bit analogue-to-digital converter (Direc Physiologic Recording System, Raytech Instruments). Maximal inspiratory pressure $(P I, \max )$ was measured at the mouth starting from functional residual capacity. Mouth occlusion pressure $\left(P_{0.1}\right)$ was measured using a one-way valve (Hans-Rudolph, Kansas City, MA, USA) connected to an electrically driven occlusion valve attached to the inspiratory line [9]. P0.1 was used to calculate the effective respiratory impedance $\left(P_{0.1} / V_{\mathrm{T}} / t \mathrm{I}\right)$ [10], and a noninvasive tension-time index of inspiratory muscles (TTmus) [11].

\section{Dyspnoea and exercise tolerance}

Dyspnoea at baseline was assessed using the Baseline Dyspnoea Index (BDI) [12]. Changes in impairment from baseline were measured using the Transition Dyspnoea Index (TDI) [12]. The BDI scores ranged from 0-12 points, the higher value corresponding to the lower dyspnoea rating. The TDI scores can vary between -9-9 points, the more positive the change, the greater the improvement. Although recent data indicate than a 1-unit increase in TDI could be clinically meaningful [13], a 2-point increase has probably a lower risk of false positive responses [14]. Exercise capacity was assessed by the 6-min walking distance (6MWD) test [15], while patients breathed room air. Dyspnoea exhibited during the walking 
tests was assessed using the Borg scale [16]. A mean increase of $54 \mathrm{~m}$ was considered to represent a clinically significant change in 6MWD in COPD patients [17].

\section{Statistical analysis}

The results were expressed as mean $\pm S D$ unless otherwise stated. Baseline values of experimental groups were compared using unpaired t-test. Chi-squared tests were used to evaluate categorical variables. The outcomes were analysed in terms of changes from baseline. Analysis of covariance (ANCOVA) was used to estimate differences in change between the experimental and sham group, with baseline values as the covariate along with group allocation [18]. Two-way analysis of variance for repeated measurements was applied to weekly changes from baseline to examine the interaction between time and treatment. In case of significant treatment-time interaction, i.e. differences between groups across time, post hoc analyses for between-group comparisons were carried out with unpaired $t$ tests at each time point. The relationships between variables were analysed using Pearson ( $\mathrm{r}$ ) and Spearman rank (rho) correlations. Statistical significance was defined as a two-tailed $\mathrm{p}<0.05$.

\section{RESULTS}

\section{Run-in period and baseline measurements}

From the 50 patients enrolled in the study, eight who showed a spontaneous reduction in $\mathrm{Pa}, \mathrm{CO}_{2} \geqslant 0.399 \mathrm{kPa}(3 \mathrm{mmHg})$ or to a value $<6.65 \mathrm{kPa}$ ( $50 \mathrm{mmHg}$ ) at the end of the run-in period were excluded. Among the 42 randomised patients, mean changes in FEV1 and $\mathrm{Pa}_{\mathrm{a}}, \mathrm{CO}_{2}$ during the run-in period $(5 \mathrm{~mL}$ and $-0.0532 \mathrm{kPa}(-0.4 \mathrm{mmHg}))$ were not significant. General baseline characteristics measured at the end of the run-in period, and the respiratory medication of the 27 treated and 15 control subjects are shown in table 1. Baseline and final (endof-treatment) physiological and clinical variables are displayed in table 2.

\section{Physiological effects of noninvasive mechanical ventilation} There were no significant differences between groups in baseline values. None of the parameters varied significantly after s-NIMV. During NIMV, $\mathrm{Pa}_{2}, \mathrm{CO}_{2}$ significantly decreased along with a slow and deep pattern of breathing. $\mathrm{Pa}, \mathrm{O}_{2}$ increased in proportion to $\mathrm{Pa}_{\mathrm{a}} \mathrm{CO}_{2}$ decrease. FEV1 and forced vital capacity (FVC) increased proportionally, and consequently the FEV1/FVC ratio remained constant. Effective respiratory impedance $\left(P_{0.1} / V_{\mathrm{T}} / \mathrm{t} \mathrm{I}\right)$ and inspiratory muscle loads, as reflected by TTmus, were decreased at the end of treatment. A small but significant increase in PI,max was also found.

All patients, except two in the control group, had severe hypoxaemia $\left(\mathrm{Pa}_{1} \mathrm{O}_{2}<7.315 \mathrm{kPa} ; 55 \mathrm{mmHg}\right)$ at baseline. After NIMV, 12 patients increased their $\mathrm{Pa}_{\mathrm{O}_{2}} \geqslant 7.315 \mathrm{kPa}$ $(55 \mathrm{mmHg})$, while the proportion of patients with severe hypoxaemia was unchanged after s-NIMV.

\section{Clinical effects of NIMV}

The 6MWD increased significantly in NIMV patients, with an average gain of $76 \mathrm{~m}$, along with a significant decrease in dyspnoea (Borg scale) at the end of the walking test (table 2). Figure 1 shows individual values for 6MWD changes and TDI in the left panel. An increase in $6 \mathrm{MWD} \geqslant 54 \mathrm{~m}$ was observed

\begin{tabular}{lccc} 
TABLE 1 & $\begin{array}{l}\text { Characteristics of } 27 \text { treated and } 15 \\
\text { chronic obstructive pulmonary disease patients }\end{array}$ \\
& NIMV & s-NIMV & p-value \\
\hline & $66 \pm 9$ & $69 \pm 7$ & 0.25 \\
Age yr & $4 / 23$ & $2 / 13$ & 0.89 \\
Sex female/male & $23 \pm 4$ & $23 \pm 4$ & 0.88 \\
BMI kg. m-2 & $67 \pm 28$ & $58 \pm 16$ & 0.17 \\
Smoking history & & & \\
$\quad$ pack-yrs & 27 & 15 & 1.00 \\
SABD as needed $\mathbf{n}$ & 20 & 10 & 0.61 \\
SABD q.i.d. $\mathbf{n}$ & 17 & 10 & 0.85 \\
LABD n & 15 & 9 & 0.78 \\
ICS $\mathbf{n}$ & 23 & 14 & 0.44 \\
THEO $\mathbf{n}$ & $30 \pm 8$ & $35 \pm 9$ & 0.14 \\
FEV $\%$ pred & $73 \pm 14$ & $79 \pm 17$ & 0.20 \\
FVC \% pred & $329 \pm 103$ & $380 \pm 94$ & 0.12 \\
6MWD m & $3.5 \pm 1$ & $3.3 \pm 1$ & 0.45 \\
BDI points & & &
\end{tabular}

Values are expressed as mean \pm SD unless otherwise stated. NIMV: noninvasive mechanical ventilation; s-NIMV: sham-NIMV; BMI: body mass index; SABD: short-acting bronchodilator; LABD: long-acting bronchodilator; ICS: inhaled corticosteroid; THEO: theophylline; FEV1: forced expiratory volume in one second; FVC: forced vital capacity; 6MWD: 6-min walking distance; BDI: baseline dyspnoea index.

in 19 of the 27 NIMV patients, whereas none of the control subjects reached this clinically significant level of improvement. The TDI at the end of treatment was also significantly higher in NIMV patients compared with the sham group (3.1 \pm 1.2 versus $0.07 \pm 0.7$ points, respectively; $\mathrm{p}<0.0001$ ), indicative of a decrease in dyspnoea. An increase of a minimum one point was found in all subjects and of at least two points in 23 of the 27 treated subjects. Such a level of improvement was not found in subjects from the control group. A close association between changes in 6MWD and TDI was found in the entire group, as shown in the left panel $(\mathrm{rho}=0.90 ; \mathrm{p}<0.0001)$ and with changes in FEV1, as shown in the right panel $(\mathrm{r}=0.60 ; \mathrm{p}<0.0001)$. Variations of the other physiological parameters were not associated with significant changes in 6MWD. By contrast, TDI was closely related to $\Delta P_{0.1} / V \mathrm{~T} / \mathrm{t} \mathrm{I}(\mathrm{rho}=-0.73), \Delta \mathrm{Pa}_{\mathrm{a}} \mathrm{CO}_{2}(\mathrm{rho}=-0.71)$, $\Delta P 0.1(\mathrm{rho}=-0.66), \Delta f \mathrm{R}(\mathrm{rho}=-0.64), \Delta \mathrm{TTmus}(\mathrm{rho}=-0.61), \Delta \mathrm{FVC}$ $(\mathrm{rho}=0.58)$, and $\Delta \mathrm{FEV} 1$ (rho $=0.55 ; \mathrm{p}<0.0001$ in all cases).

Time course of noninvasive mechanical ventilation effects Weekly evolution during treatment and the following 2 weeks is shown in figures 2 and 3. Figure 2 shows the pattern of breathing, arterial blood gases, and spirometry while figure 3 includes respiratory mechanics and exercise capacity. All physiological variables started to exhibit significant changes from the end of the first week of treatment, except $P_{I}$,max and TTmus, which achieved significant changes later. These effects were progressive and maximal by the end of the third week. Clinical improvements in terms of exercise capacity mirrored those physiological changes. All these changes appeared to gradually fade away from the first week after treatment 
TABLE 2 Effects of treatment on lung function, exercise capacity and dyspnoea in 15 controls and 27 treated chronic obstructive pulmonary disease patients

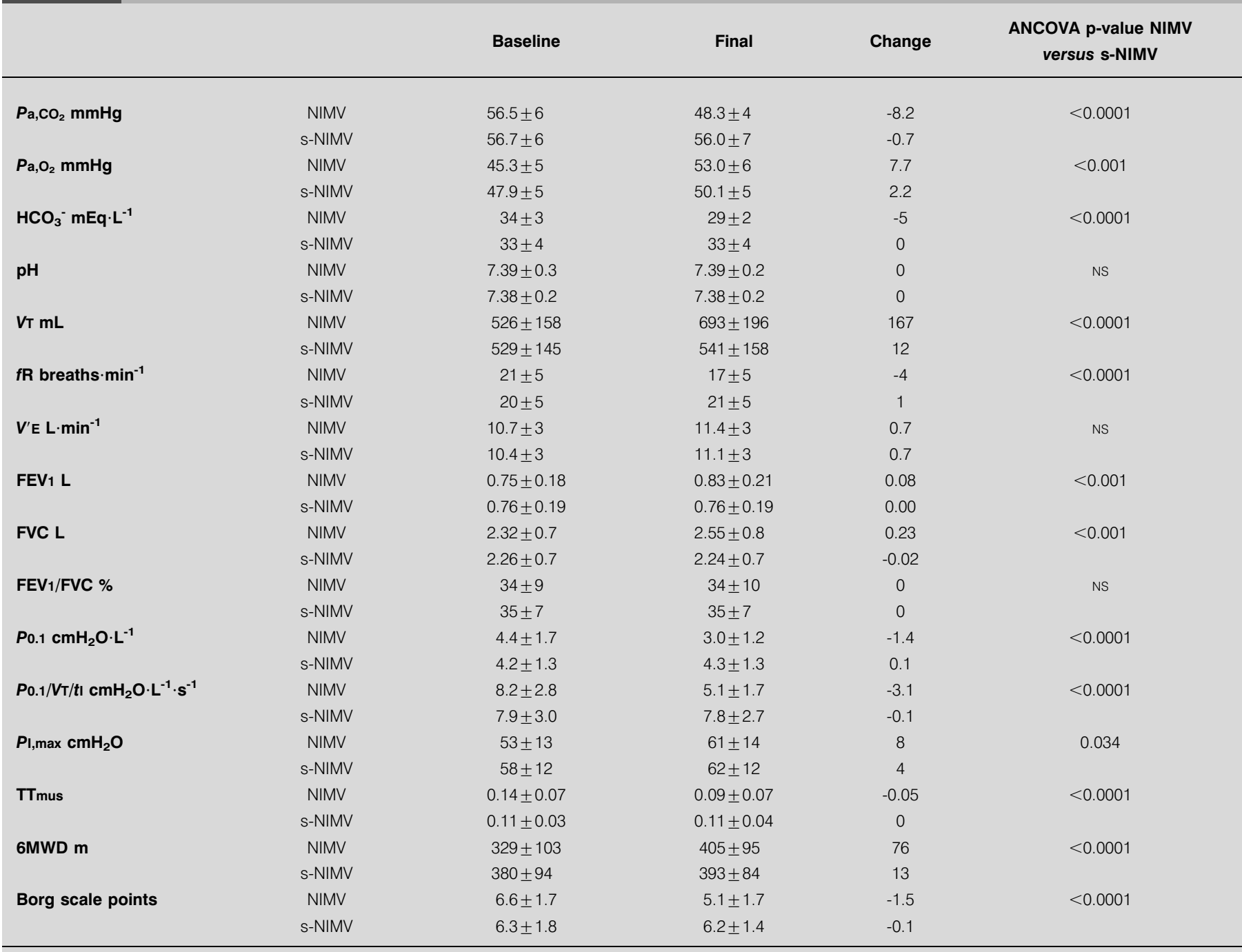

Data are expressed as mean $\pm \mathrm{SD}$. ANCOVA: analysis of covariance; NIMV: noninvasive mechanical ventilation; s-NIMV: sham-NIMV; Pa, $\mathrm{O}_{2}$ : arterial oxygen tension; $\mathrm{Pa}_{1} \mathrm{CO}_{2}$ : carbon dioxide arterial tension; $\mathrm{HCO}_{3}{ }^{-}$: serum bicarbonate; $\mathrm{VT}$ : tidal volume; $f \mathrm{R}$ : breathing frequency; $V^{\prime} \mathrm{E}$ : minute ventilation; $\mathrm{FEV} 1$ : forced expiratory volume in one second; FVC: forced vital capacity; $P_{0.1}$ : mouth occlusion pressure; $P_{0.1} / \mathrm{VT} / t \mathrm{l}$ : effective respiratory impedance; $P_{\mathrm{I} \text {,max: }}$ maximal inspiratory pressure; $T$ Tmus: tensiontime index of inspiratory muscles; 6MWD: 6-min walking distance; NS: not significant.

although most were still significant 2 weeks after cessation. At this point, $94 \%$ of the gain in $P_{\mathrm{I}}$ max and $82 \%$ of the gain in $6 \mathrm{MWD}$ were maintained, in contrast to only $31 \%$ for $\mathrm{FEV} 1,42 \%$ for $V \mathrm{~T}$ and $52 \%$ for $P_{0.1} / V \mathrm{~T} / \mathrm{tI}$.

\section{DISCUSSION}

The main findings of the present study include: 1) Diurnal short-term intermittent NIMV in stable hypercapnic COPD patients results in clinical improvement, reducing dyspnoea and enhancing exercise capacity in addition to improvement in ABG, breathing pattern and respiratory mechanics. 2) All of the physiological effects are significant within the first week of treatment, except for unloading of the inspiratory muscles which becomes significant only after 2 weeks.
Physiologial effects of noninvasive mechanical ventilation Improvements in $\mathrm{Pa}_{1} \mathrm{CO}_{2}$ have been previously reported after short-term application of NIMV using either negative or positive pressure ventilation. AMBROsINO et al. [19] reported a reduction of $\mathrm{Pa}_{\mathrm{a}} \mathrm{CO}_{2}$ after only two sessions of NIMV. A slow and deep pattern of breathing has been reported by SCANO et al. [20] after 7 days of ventilation and after 4 weeks by others [21, 22]. Interestingly, GigLIOTTI et al. [21] measured the sequence of changes in $\mathrm{Pa}_{1} \mathrm{CO}_{2}$ and $V \mathrm{~T}$ after 13 and 27 days of ventilation and found that improvement was progressive. All of the above studies have concluded that beneficial effects of NIMV can be attributed to the improvement in inspiratory muscle function, particularly because the rest achieved during ventilation sessions could alleviate a possible "chronic fatigue" of the 

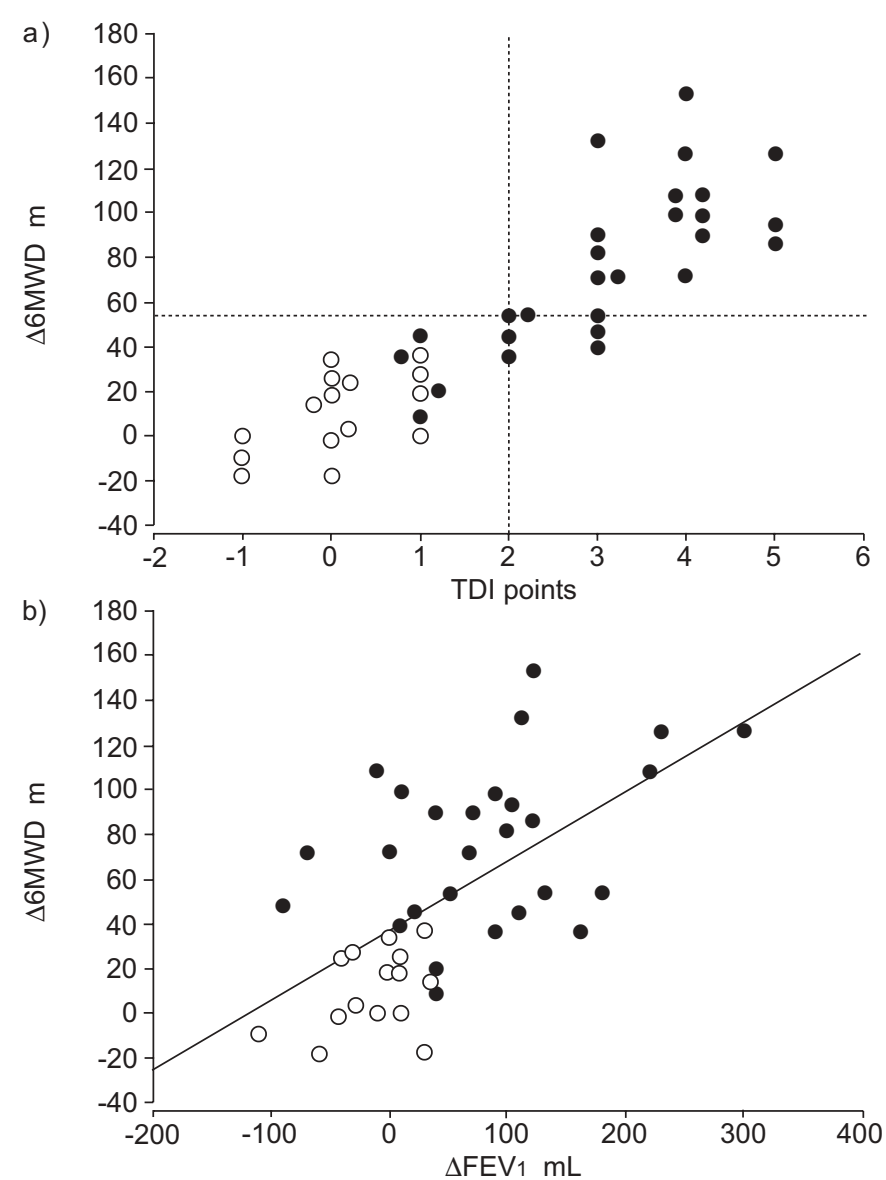

FIGURE 1. Relationship between: a) changes from baseline $(\Delta)$ in the 6-min walking distance (6MWD) and transition dyspnoea index (TDI; rho=0.90; $p<0.0001$ ), where dotted lines represent clinically meaningful improvements for each parameter; and b) $\triangle 6 \mathrm{MWD}$ and $\Delta$ forced expiratory volume in one second $(\Delta \mathrm{FEV} 1)$, with regression line $\left(\Delta 6 \mathrm{MWD}: 38+0.3 \times \Delta \mathrm{FEV}_{1} ; \mathrm{r}=0.60 ; \mathrm{p}<0.0001\right)$. - : noninvasive mechanical ventilation (NIMV); $O$ : sham-NIMV.

inspiratory muscles. Clearly, the present study does not support this hypothesis, since the baseline TTmus values were found to be approximately half the critical level associated with inspiratory muscle fatigue [11]. In addition, PI,max increased significantly only at the end of the treatment period, after all other changes had taken place.

Why did these patients reduce $\mathrm{Pa}_{1} \mathrm{CO}_{2}$ so quickly after NIMV? The current authors have previously shown that tidal volume was markedly increased during NIMV sessions [3], and the present study shows that a deep and slow pattern of breathing is maintained by the patients between sessions. Such pattern of breathing has been shown to reduce $\mathrm{Pa}_{1} \mathrm{CO}_{2}$ in hypercapnic COPD patients but it has been reported that it markedly increases the work of breathing and induces fatigue [23]. TTmus analysis shows that this was not the case in the patients in the present study, the current authors suggest that the effect may be explained by the observed lower effective respiratory impedance. Indeed, the patients could not only achieve higher levels of alveolar ventilation with no additional energetic cost during the first week of treatment, but they actually decreased their TTmus when effective respiratory impedance further improved. The current authors previously demonstrated that lung volume deflation was a key factor to explain these benefits [3], as also shown with the use of long-acting bronchodilators [24, 25]. The effect of NIMV on spirometric lung volumes, dyspnoea, and exercise tolerance cannot be attributed to the use of bronchodilators, since the latter was similarly used in the two groups. However, NIMV clearly provides further benefits to patients on appropriate bronchodilation therapy.

\section{Clinical effects of noninvasive mechanical ventilation}

In the present study, the authors were particularly interested in the clinical effects of NIMV. Therefore, the increase in $6 \mathrm{MWD}$ was impressive as it had only been previously reported in uncontrolled trials [19, 21, 26]. RENSTON et al. [27] found an increase in the $6 \mathrm{MWD}$ after 5 days of ventilation. Improvements in exercise capacity were also reported in other short- [19, 21] and long-term studies [26]. In contrast, randomised controlled trials have failed to show a significant improvement in the 6MWD [5, 28-31]. There are several possible reasons to explain the discrepancy. First, in most of the previous studies the amount of inspiratory pressure was lower than that employed in the present study, being similar only in the study of MEECHAM JONES et al. [5]. It has been shown that high inspiratory pressures can modify the breathing pattern, increasing $V \mathrm{~T}$ and $t \mathrm{E}[3,32]$, providing room to reduce $\mathrm{Pa}_{\mathrm{a}} \mathrm{CO}_{2}$ and decrease lung hyperinflation. The current authors found in this and in a previous study [3] that progressive increase to high inspiratory pressures is possible with daytime supervision. It should be noted that supervision of the procedure is limited when NIMV is applied at home, and consequently compliance is reduced because of mask leaks, asynchrony between the patient and ventilator, and discomfort [33]. Finally, as pointed out by WIJKSTRA et al. [34] the high upper limit of the confidence interval for the 6MWD $(82 \mathrm{~m})$ found in the randomised controlled trials suggests that at least some patients might improve their exercise capacity after NIMV. The present data are in agreement with the hypothesis, as only $70 \%$ of the patients submitted to NIMV (figure 1) increased the $6 \mathrm{MWD} \geqslant 54 \mathrm{~m}$. It is noteworthy that the improvement in the 6MWD after the 2 week follow-up was maintained to a greater extent than any of the physiological changes. A possible explanation for this finding is that these patients could have increased their daily physical activities during the treatment period, achieving some degree of general training.

It is also of interest that some reduction in dyspnoea was found in all treated subjects and that this change was a far better predictor of exercise capacity than any single physiological parameter. Improvement in dyspnoea after NIMV had been reported in two randomised controlled trials [30,31]. However, these studies did not suggest a mechanism for the alleviation of dyspnoea. The current data shows that it is related to a reduction in inspiratory loads (decrease in $P_{0.1} / V_{\mathrm{T}} / \mathrm{t}_{\mathrm{I}}$ and TTmus), and to a slower breathing rate which promotes lung deflation.

An unexpected finding was the high proportion of treated patients (12 out of 27) that increased their $\mathrm{Pa}_{\mathrm{a}_{2}} \mathrm{O}_{2}>7.315 \mathrm{kPa}$ (55 $\mathrm{mmHg}$ ) by the end of treatment. It can be speculated that 

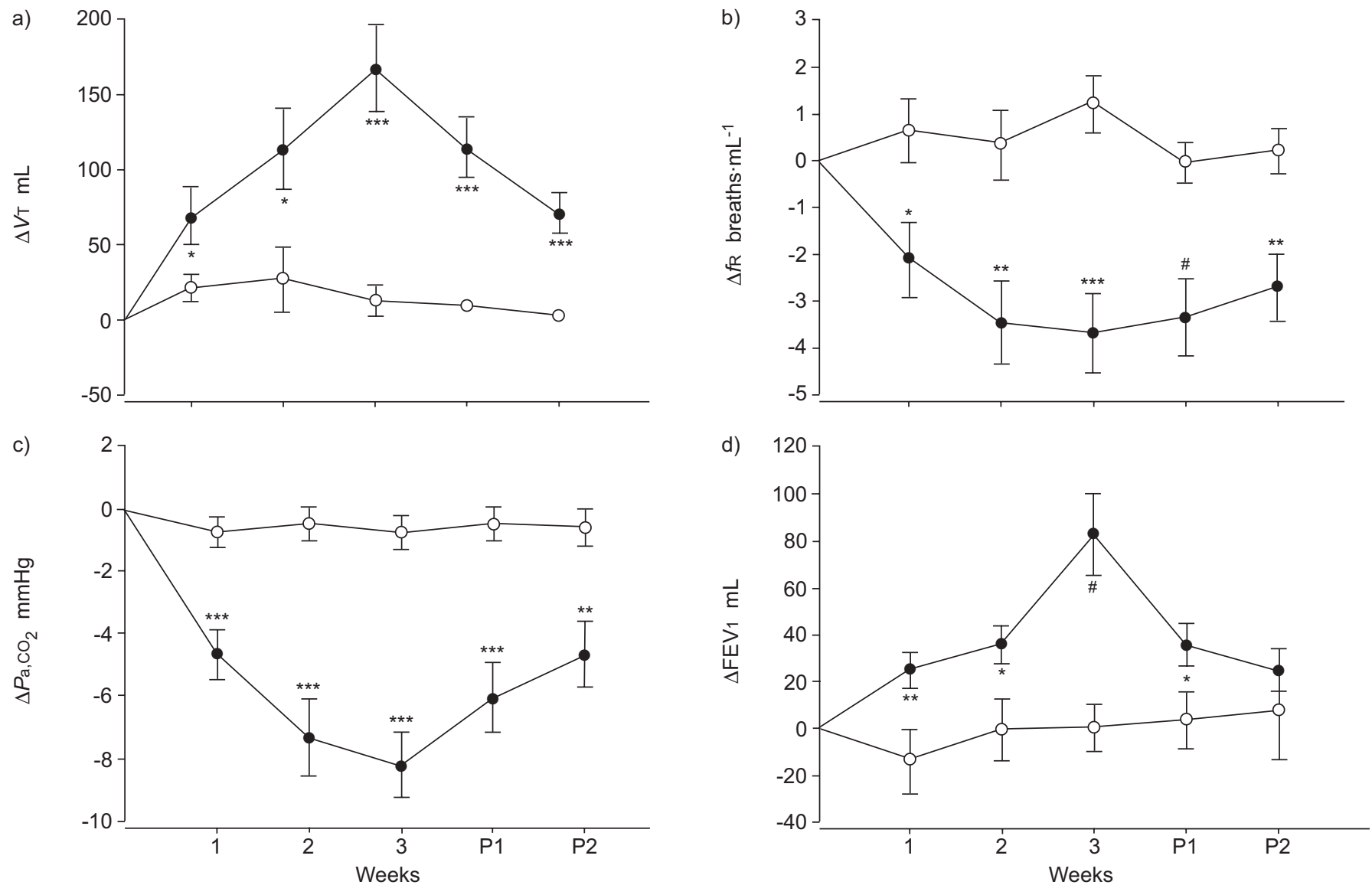

FIGURE 2. Changes from baseline $(\Delta)$ in: a) tidal volume $(\mathrm{VT})$; b) breathing frequency $(f R)$; c) carbon dioxide arterial tension $\left(P a, \mathrm{CO}_{2}\right)$; and d) forced expiratory volume in one second (FEV1). - noninvasive mechanical ventilation (NIMV); O: sham-NIMV. Weeks 1, 2, and 3 refer to end of weeks 1,2 and 3 of treatment, whereas P1 and P2 indicate weeks 1 and 2 of follow-up. Values are expressed as mean \pm SEM. ${ }^{*}: p<0.05 ;{ }^{* *}: p<0.01 ;{ }^{* *}: p<0.001 ;{ }^{*}: p<0.0001$ for between-group comparisons

some of these patients may no longer need long-term $\mathrm{O}_{2}$ therapy, resulting in a significant gain in quality of life.

\section{Clinical implications}

NIMV may be an effective component of pulmonary rehabilitation in severe hypercapnic COPD patients. However, the best schedule to deliver NIMV in this condition is not yet known. In the present study, NIMV was applied at the hospital during the day in outpatients in order to achieve adequate supervision of the procedure. Diurnal application of NIMV is not widely accepted, the common practice being to apply it at home overnight during sleep [5, 28-31]. The main reason for night time application comes from studies which demonstrate that benefits obtained with NIMV are related to an improvement in sleep-related hypoventilation and sleep architecture [5, 35]. However, in a group of patients with extra pulmonary restrictive disease, SCHONHOFER et al. [36] showed that NIMV was equally effective in improving sleep characteristics when administered during the day or overnight. Furthermore, there is no study comparing the efficacy of home versus hospital application of NIMV or addressing the importance of supervision. The present study provides evidence supporting its daytime use, in line with previous studies in which negative pressure ventilators were employed [18-20, 26, 37]. Daytime use of short term NIMV in the hospital setting allowed a closer supervision of treatment and insured patient compliance. Although patient compliance with this protocol, in the long term, is not known and widespread use may result in significant hospital burden, the present study suggests that an active supervision of treatment, at least in the first weeks after initiating NIMV, is a major factor for success. The current data also suggest that NIMV need not be applied as frequently, as residual effects were found up to 2 weeks after the end of treatment. Residual effects of NIMV have been previously reported by FERNANDEZ et al. [37] and could also help to explain the results of GUTIÉRREZ et al. [26] who have found that applying NIMV once a week provides benefits to hypercapnic COPD patients. A less frequent daytime application might prove useful and improve adherence to NIMV, reducing the high dropout rate found in some previous studies [28, 29].

To summarise from a clinical standpoint, the present study supports daytime use of noninvasive mechanical ventilation, as previously shown with negative pressure ventilators. It also suggests that noninvasive mechanical ventilation does not have to be applied on a daily basis, since residual physiological effects and improvement in exercise capacity were found up to 2 weeks after cessation. Both diurnal and intermittent application of noninvasive mechanical ventilation could make this treatment more tolerable for patients, with fewer dropout rates. 

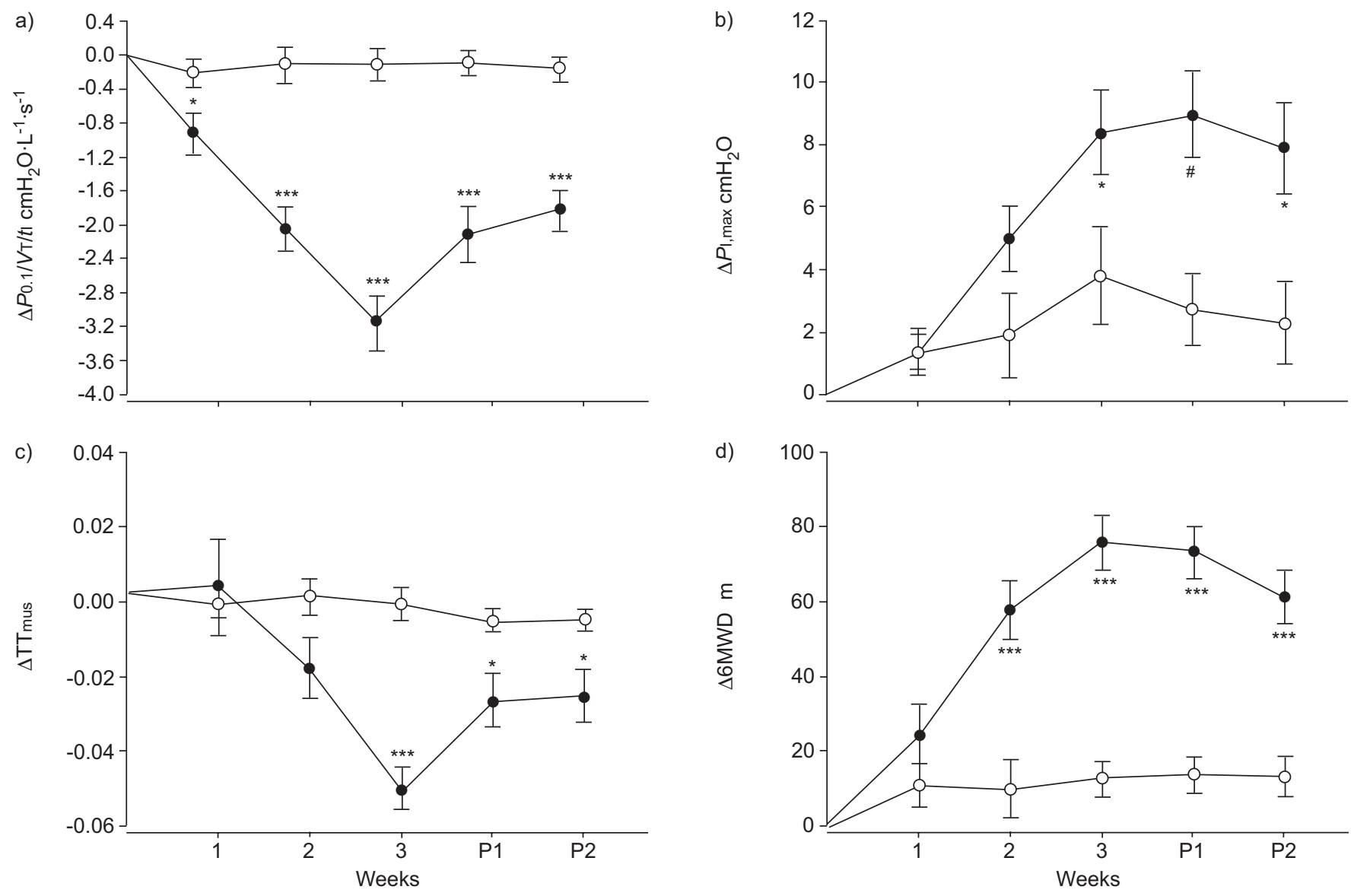

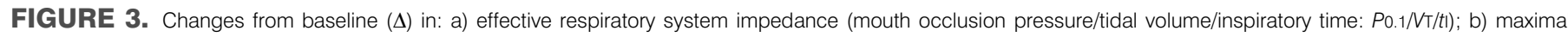
inspiratory pressure ( $\mathrm{PI}, \mathrm{max})$; c) tension time index of inspiratory muscles (TTmus); and d) 6-min walking distance (6MWD). $\bullet$ : noninvasive mechanical ventilation (NIMV); $O$ : sham-NIMV. Weeks 1,2, and 3 refer to end of weeks 1,2 and 3 of treatment, whereas P1 and P2 indicate weeks 1 and 2 of follow-up. Values are expressed as mean \pm SEM. *: $p<0.05 ;{ }^{* *}: \mathrm{p}<0.001 ;{ }^{*}: \mathrm{p}<0.0001$ for between-group comparisons

\section{REFERENCES}

1 Brochard L, Mancebo J, Elliott MW. Noninvasive ventilation for acute respiratory failure. Eur Respir J 2002; 19: 712-721.

2 Elliot MW. Noninvasive ventilation in chronic ventilatory failure due to chronic obstructive pulmonary disease. Eur Respir J 2002; 20: 511-514.

3 Díaz O, Bégin P, Torrealba B, Jover E, Lisboa C. Effects of nonivasive ventilation on lung hyperinflation in stable hypercapnic COPD. Eur Respir J 2002; 20: 1490-1498.

4 Elliott MW, Mulvey DA, Moxham J, Green M, Branthwaite MA. Domiciliary nocturnal nasal intermittent positive pressure ventilation in COPD: mechanisms underlying changes in arterial blood gas tensions. Eur Respir J 1991; 4: 1044-1052.

5 Meecham Jones DJ, Paul EA, Jones PW, Wedzicha JA. Nasal pressure support ventilation plus oxygen compared with oxygen therapy alone in hypercapnic COPD. Am J Respir Crit Care Med 1995; 152: 538-544.

6 Celli BR, W, MacNee W, Agusti A, et al. ATS/ERS Task Force. Standards for the diagnosis and treatment of patients with COPD: a summary of the ATS/ERS position paper. Eur Resp J 2004; 23: 932-946.
7 American Thoracic Society. Standardization of spirometry: 1994 update. Am J Respir Crit Care Med 1995; 152: 1107-1136

8 Knudson RJ, Lebowist MD, Holberg CI, Burrows B. Changes in the normal maximal expiratory flow-volume curve with growth and age. Am Rev Respir Dis 1983; 127: 725-734.

9 Whitelaw WA, Derenne JP, Milic-Emili J. Occlusion pressure as a measure of respiratory center output in conscious man. Respir Physiol 1975; 23: 181-199.

10 Hussain S, Pardy R, Dempsey J. Mechanical impedance as determinant of inspiratory neural drive during exercise in humans. J Appl Physiol 1985; 59: 365-375.

11 Ramonatxo M, Boulard P, Prefaut C. Validation of a noninvasive tension-time index of inspiratory muscles. $J$ Appl Physiol 1995; 78: 646-653.

12 Mahler DA, Weinberg DH, Wells CK, Feinstein AR. The measurement of dyspnea. Contents, interobserver agreement, and physiologic correlates of two new clinical indexes. Chest 1984; 85: 751-758.

13 Witek TJ, Mahler DA. Minimal important difference of the transition dyspnoea index in a multinational clinical trial. Eur Respir J 2003; 21: 267-272. 
14 Reardon J, Awad E, Normandin E, Vale F, Clark B, ZuWallak RL. The effect of comprehensive outpatient pulmonary rehabilitation on dyspnea. Chest 1994; 105: 1046-1052.

15 Butland RJA, Pang J, Gross ER, Woodcock AA, Geddes DM. Two, six and twelve-minute walking tests in respiratory diseases. BMJ 1982; 284: 1607-1608.

16 Borg GAV. Psychophysical basis of perceived exertion. Med Sci Sports Exerc 1982; 14: 377-381.

17 Redelmeier DA, Bayoumi AM, Goldstein RS, Guyatt GH. Interpreting small differences in functional status: the six minute walk test in chronic lung disease patients. Am J Respir Crit Care Med 1997; 155: 1278-1282.

18 Vickers AJ, Altman DG. Analysing controlled trials with baseline and follow up measurements. BMJ 2001; 323 : 1123-1124.

19 Ambrosino N, Montagna T, Nava S, et al. Short term effect of intermittent negative pressure ventilation in COPD patients with respiratory failure. Eur Respir J 1990; 3: 502-508.

20 Scano G, Gigliotti F, Duranti R, Spinelli A, Gorini M, Schiavina M. Changes in ventilatory muscle function with negative pressure ventilation in patients with severe COPD. Chest 1990; 97: 322-327.

21 Gigliotti F, Spinelli A, Duranti R, Gorini M, Goti P, Scano G. Four-week negative pressure ventilation improves respiratory function in severe hypercapnic COPD patients. Chest 1994; 105: 87-94.

22 Nava S, Fanfulla F, Frigerio P, Navalesi P. Physiologic evaluation of 4 weeks of nocturnal nasal positive pressure ventilation in stable hypercapnic patients with chronic obstructive pulmonary disease. Respiration 2001; 68: 573-583.

23 Bellemare F, Grassino A. Force reserve of the diaphragm in patients with chronic obstructive pulmonary disease. J Appl Physiol 1983; 55: 8-15.

24 O'Donnell DE, Voduc N, Fitzpatrick M, Webb KA. Effect of salmeterol on the ventilatory response to exercise in chronic obstructive pulmonary disease. Eur Respir J 2004; 24: 86-94.

25 O'Donnell DE, Flüge T, Gerken F, et al. Effects of tiotropium on lung hyperinflation, dyspnoea and exercise tolerance in COPD. Eur Respir J 2004; 23: 832-840.

26 Gutiérrez M, Beroíza T, Contreras G, et al. Weekly cuirass ventilation improves blood gases and inspiratory muscle function in patients with chronic air-flow limitation and hypercarbia. Am Rev Respir Dis 1988; 138: 617-623.

27 Renston JP, DiMarco AF, Supinsky GS. Respiratory muscle rest using nasal BiPAP ventilation in patients with stable severe COPD. Chest 1994; 105: 1053-1060.

28 Strumpf DA, Millman RP, Carlisle CC, et al. Nocturnal positive-pressure ventilation via nasal mask in patients with severe chronic obstructive pulmonary disease. Am Rev Respir Dis 1991; 144: 1234-1238.

29 Gay PC, Hubmayr RD, Stroetz RW. Efficacy of nocturnal nasal ventilation in stable, severe chronic obstructive pulmonary disease during a 3-month controlled trial. Mayo Clin Proc 1996; 71: 533-42.

30 Casanova C, Celli B, Tost L, et al. Long-term controlled trial of nocturnal nasal positive pressure ventilation in patients with severe COPD. Chest 2000; 118: 1582-90.

31 Clini E, Sturani C, Rossi A, et al. The Italian multicentre study on noninvasive ventilation in chronic obstructive pulmonary disease patients. Eur Respir J 2002; 20: 529-538.

32 Nava S, Ambrosino N, Rubini F, et al. Effect of nasal pressure support ventilation and external PEEP on diaphragmatic activity in patients with severe stable COPD. Chest 1993; 103: 143-150.

33 Criner GJ, Brennan K, Travaline JM, Kreimer D. Efficacy and compliance with noninvasive positive pressure ventilation in patients with chronic respiratory failure. Chest 1999; 116: 667-675.

34 Wijkstra PJ, Lacasse Y, Guyatt GH, et al. A meta-analysis of nocturnal noninvasive positive pressure ventilation in patients with stable COPD. Chest 2003; 124: 337-343.

35 Elliot MW, Simonds AK, Carroll MP, Wedzicha JA, Branthwaite MA. Domiciliary nocturnal nasal intermittent positive pressure ventilation in hypercapnic respiratory failure due to chronic obstructive lung disease: effects on sleep and quality of life. Thorax 1992; 47: 342-348.

36 Schonhofer B, Geibel M, Sonneborn M, Haidl P, Kohler D. Daytime mechanical ventilation in chronic respiratory insufficiency. Eur Respir J 1997; 10: 2840-46.

37 Fernandez E, Weiner P, Meltzer E, Lutz MM, Badish DB, Cherniack RM. Sustained improvement in gas exchange after negative pressure ventilation for 8 hours per day on 2 successive days in chronic airflow limitation. Am Rev Respir Dis 1991; 144: 390-394. 